\title{
$\mathbf{m} / \mathbf{S}$
}

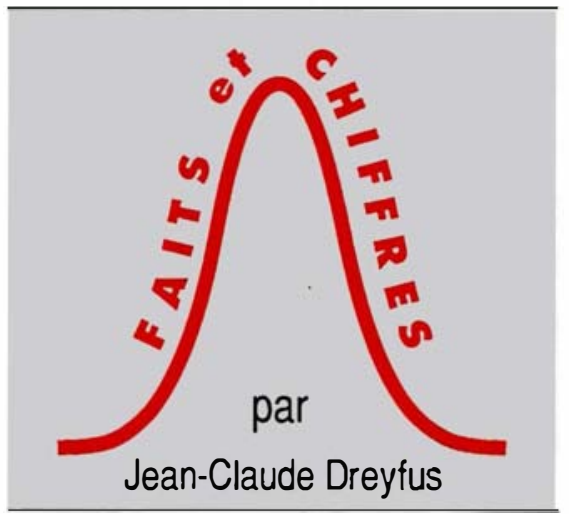

$P$ armi les paramètres du génome, l'un des plus importants est sa taille, et celle de ses constituants, les chromosomes. Newton E. Morton (Southampton, GB) fait le point [1] sur les données actuelles, qui ne sont sans doute pas définitives. On distingue deux cartes du génome, physique et génétique. Pour la carte physique, le contenu d'un chromosome est mesuré avec la meilleure précision en tant que pourcentage du génome total. Pour transformer ce pourcentage en longueur, exprimée en mégabases $(\mathrm{Mb})$, il faut connaître la taille totale du génome haploïde, estimée à $3200 \mathrm{Mb}$, correspondant à 3,5 picogrammes. Les principales méthodes incluent la cytométrie de flux ou d'image et l'autoradiographie. Les cartes génétiques se fondent sur des données cytogénétiques et de liaisons génétiques qui laissent encore place à des approximations. On le sait, la taille génétique diffère selon le sexe, celle de la femme étant beaucoup plus étendue que celle de l'homme $\left(\mathrm{m} / \mathrm{s} n^{\circ} 4\right.$, vol. 7, p. 392). Enfin, l'analyse peut

1. Morton NE. Parameters of the human genome. Proc Natl Acad Sci USA 1991; 88 : 7474-6.

$\mathrm{m} / \mathrm{s} n^{\circ} 9$, vol. 7, novembre 91

\section{La taille des chromosomes humains} chromosomes humains

\begin{tabular}{|c|c|c|c|}
\hline \multicolumn{4}{|c|}{$\begin{array}{l}\text { Tableau I } \\
\text { OUE ET GÉNÉTIQUE DES BRAS } \\
\text { OMOSOMES HUMAINS } \\
\text { du tableau, p. 964) }\end{array}$} \\
\hline \multirow[t]{2}{*}{ Chromosome } & \multirow{2}{*}{$\begin{array}{c}\text { Longueur physique } \\
\text { (Mb) }\end{array}$} & \multicolumn{2}{|c|}{ Longueur génétique (cM) } \\
\hline & & homme & femme \\
\hline $1 p$ & 128 & 106 & 189 \\
\hline $1 q$ & 135 & 104 & 188 \\
\hline $2 p$ & 99 & 83 & 152 \\
\hline $2 q$ & 156 & 105 & 192 \\
\hline $3 p$ & 99 & 80 & 106 \\
\hline $3 q$ & 115 & 104 & 136 \\
\hline $4 p$ & 56 & 52 & 117 \\
\hline $4 q$ & 147 & 94 & 213 \\
\hline $5 p$ & 52 & 53 & 93 \\
\hline $5 q$ & 142 & 101 & 175 \\
\hline $6 p$ & 65 & 57 & 110 \\
\hline $6 q$ & 118 & 87 & 166 \\
\hline $7 p$ & 65 & 60 & 81 \\
\hline $7 q$ & 106 & 91 & 124 \\
\hline $8 p$ & 50 & 50 & 75 \\
\hline $8 q$ & 105 & 88 & 131 \\
\hline $9 p$ & 51 & 50 & 64 \\
\hline $9 q$ & 94 & 79 & 81 \\
\hline $10 p$ & 44 & 54 & 81 \\
\hline $10 q$ & 100 & 84 & 129 \\
\hline $11 p$ & 58 & 53 & 84 \\
\hline $11 \mathrm{q}$ & 86 & 63 & 100 \\
\hline $12 p$ & 39 & 45 & 80 \\
\hline $12 q$ & 104 & 84 & 148 \\
\hline $13 p$ & 16 & 0 & 0 \\
\hline $13 q$ & 98 & 100 & 160 \\
\hline $14 p$ & 16 & 0 & 0 \\
\hline $14 q$ & 93 & 104 & 131 \\
\hline $15 p$ & 17 & 0 & 0 \\
\hline $15 q$ & 89 & 102 & 199 \\
\hline $16 p$ & 39 & 60 & 96 \\
\hline $16 q$ & 59 & 56 & 90 \\
\hline $17 p$ & 28 & 51 & 126 \\
\hline $17 q$ & 64 & 66 & 161 \\
\hline $18 p$ & 20 & 50 & 76 \\
\hline $18 q$ & 65 & 63 & 97 \\
\hline
\end{tabular}




\begin{tabular}{|c|c|c|c|}
\hline \multicolumn{4}{|c|}{$\begin{array}{l}\text { Tableau I (suite) } \\
\text { YSIQUE ET GÉNÉTIQUE DES BRAS } \\
\text { HROMOSOMES HUMAINS }\end{array}$} \\
\hline \multirow[t]{2}{*}{ Chromosome } & \multirow{2}{*}{$\begin{array}{l}\text { Longueur physique } \\
(\mathrm{Mb})\end{array}$} & \multicolumn{2}{|c|}{ Longueur génétique (cM) } \\
\hline & & homme & femme \\
\hline $\begin{array}{l}19 p \\
19 q \\
20 p \\
20 q \\
21 p \\
21 q \\
22 p \\
22 q \\
X p \\
X q \\
Y p \\
Y q \\
\text { Autosomes }\end{array}$ & $\begin{array}{c}30 \\
37 \\
31 \\
41 \\
11 \\
39 \\
13 \\
43 \\
62 \\
102 \\
13 \\
46 \\
3063\end{array}$ & $\begin{array}{r}50 \\
50 \\
42 \\
42 \\
0 \\
76 \\
0 \\
70 \\
50 \\
0 \\
50 \\
0 \\
2809\end{array}$ & $\begin{array}{r}97 \\
97 \\
109 \\
109 \\
0 \\
108 \\
0 \\
92 \\
87 \\
133\end{array}$ \\
\hline
\end{tabular}

être raffinée en mesurant séparément la longueur des deux bras pour chaque chromosome. Le Tableau I (cicontre et p. 980) reproduit ces données qui sont les plus détaillées. Il est aisé d'en déduire la taille globale de chacun des chromosomes, ainsi que la moyenne des deux sexes pour la carte génétique. Le lecteur pourra s'étonner de voir que pour plusieurs autosomes $(13,14,15,21,22)$ la taille du bras court soit notée 0 dans la carte génétique. C'est parce que, dans ces chromosomes acrocentriques, la taille du bras court est très réduite, qu'on n'y connaît aucune recombinaison, et qu'on n'y a localisé que des gènes d'ARN ribosomique. La connaissance de ces paramètres est importante pour les interprétations précises des résultats que fournit le projet d'étude du génome humain 